\title{
Remerciements aux relecteurs 2019
}

\section{Thanks to 2019 reviewers}

La revue Cahiers Agricultures remercie tous les relecteurs qui ont bien voulu l'aider à prendre une décision sur la publication des textes et ont fourni aux auteurs de précieux conseils.

\author{
Aboulfaraj, Said \\ Adamczewski, Amandine \\ Andreas, Christophe \\ Andrieu, Nadine \\ Angeon, Valérie \\ Ankogui-Mpoko, Guy-Florent \\ Araujo-Bonjean, Catherine \\ Aubert, Magali \\ Audouin, Sarah \\ Augusseau, Xavier \\ Barreteau, Olivier \\ Barrey, Sandrine \\ Bassett, Thomas \\ Bazile, Didier \\ Bédrani, Slimane \\ Bégué, Agnès \\ Bélières, Jean-François \\ Berti, Fabio \\ Brondeau, Florence \\ Bryant, Christopher \\ Carimentrand, Aurélie \\ Casabianca, François \\ Cerdan, Claire \\ Champredonde, Marcelo \\ Chauvin, Emmanuel \\ Cognard, Françoise \\ Collard, Anne-Laure \\ Cournut, Sylvie \\ Couture, Jean-Louis \\ Coyne, Danny \\ Dagno, Karim \\ Danthu, Pascal \\ De Bon, Hubert \\ Del Vecchio, Kevin \\ Demenois, Julien \\ Desquilbet, Marion \\ Djamen, Patrice \\ Drogué, Sophie \\ Droy, Isabelle \\ Ducroquet, Hubert \\ Dugué, Patrick \\ Duvernoy, Isabelle \\ Ehrlich, Marianne
}

Faure, Jean-François

Feder, Frédéric

Ferchaud, Fabien

Fernandes, Paula

Fok, Michel

Gaillard, Cédric

Gautier, Denis

Gazull, Laurent

Ghiotti, Stéphane

Gonin, Alexis

Gouda, Abdoul-Ibrachi

Guibert, Hervé

Hartani, Tarik

Havard, Michel

Huat, Joël

Huguenin, Johann

Jacob, Jean-Pierre

Jamin, Jean-Yves

Jeanneaux, Philippe

Koubaa, Yamen

Lacombe, Guillaume

Lardon, Sylvie

Lauvie, Anne

Le Bellec, Fabrice

Lebailly, Philippe

Lebourgeois, Valentine

Lejars, Caroline

Lida, Serge

Livian, Yves

Mariel, Juliette

Marie-Vivien, Delphine

Marsden, Claire

Martin, Thibaud

Mayaux, Pierre-Louis

Mazé, Armelle

Mbetid-Bessane, Emmannuel

Michel, Isabelle

Mokrini , Fouad

Mollard, Eric

Mougenot, Isabelle

Moustier, Paule

Mundler, Patrick

Naudin, Krishna
Navarrete, Mireille

Oumarou, Amadou

Peigne, Joséphine

Pichot, Jean-Pascal

Raharison, Tahina

Rasplus, Valery

Rawski, Christine

Ricau, Pierre

Richard, Frédéric

Ruf, François

Sabourin, Eric

Saint-Macary, Camille

Salliou, Nicolas

Sanon, Antoine

Sarthou, Jean-Pierre

Sautier, Denis

Sautier, Marion

Serpantié, Georges

Silvie, Pierre

Simon, Serge

Sokona, Fatoumata

Son, Diakalia

Sorba, Jean-Michel

Soulard, Christophe

Sraïri, Mohamed

Tallon, Hélène

Tarrouth, Gabin

Temple, Ludovic

Thonart, Philippe

Tondoh Ebagnerin, Jérôme

Tonneau, Jean-Philippe

Toulet, Anne

Tournebize, Régis

Touzard, Jean-Marc

Trouche, Gilles

Turlot, Amélie

Vaksmann, Michel

Valette, Elodie

Vall, Eric

Vernier, Philippe

Zakhia-Rozis, Nadine

Zampaligre, Nouhoun

Zoundi Sibiri, Jean 